\title{
BMJ Open How do potentially inappropriate medications and polypharmacy affect mortality in frail and non-frail cognitively impaired older adults? A cohort study
}

\author{
Bryony Porter, ${ }^{1}$ Antony Arthur, $^{2}$ George M Savva ${ }^{1,3}$
}

To cite: Porter B, Arthur A, Savva GM. How do potentially inappropriate medications and polypharmacy affect mortality in frail and non-frail cognitively impaired older adults? A cohort study. BMJ Open 2019;9:e026171. doi:10.1136/ bmjopen-2018-026171

- Prepublication history and additional material for this paper are available online. To view these files, please visit the journal online (http://dx.doi. org/10.1136/bmjopen-2018026171).

Received 10 September 2018 Revised 14 December 2018 Accepted 18 February 2019

A) Check for updates

(C) Author(s) (or their employer(s)) 2019. Re-use permitted under CC BY-NC. No commercial re-use. See rights and permissions. Published by BMJ.

${ }^{1}$ School of Health Sciences, University of East Anglia, Norwich, UK

${ }^{2}$ School of Health Sciences, University of East Anglia,

Norwich, UK

${ }^{3}$ Quadram Institute Bioscience, Norwich, UK

Correspondence to

Dr George M Savva;

george.savva@quadram.ac.uk

\section{ABSTRACT}

Objectives To test whether the use of potentially inappropriate central nervous system acting medications, proton pump inhibitors (PPIs) or polypharmacy are associated with mortality in cognitively impaired older adults and whether frailer people are at greater risk of harm.

Setting A cohort study nested within the Cognitive Function and Ageing Study II, a population representative cohort study of the older population in Cambridgeshire, Nottingham and Newcastle, UK.

Participants A total of 1154 cognitively impaired participants, aged 65 years or older.

Exposures Any use of antipsychotics, antidepressants, other anticholinergic medication, benzodiazepines or PPIs, polypharmacy (5-9) and hyperpolypharmacy ( $\geq 10$ reported medications) were ascertained at baseline. Frailty was assessed using the Fried criteria.

Primary outcome Mortality up to 8 years follow-up. HRs associated with potentially inappropriate medication (PIM), frailty and their interaction were estimated adjusting for covariates.

Results Within the sample, $44 \%$ were taking one or more PIM. Apart from antipsychotics (adjusted HR=3.24, 95\% Cl 1.83 to 5.73), use of specific PIM was not associated with greater subsequent mortality. Polypharmacy $(\mathrm{HR}=1.17,95 \% \mathrm{Cl} 0.95$ to 1.45$)$ and hyperpolypharmacy were associated with mortality ( $\mathrm{HR}=1.60,95 \% \mathrm{Cl} 1.16$ to 2.22). Being frail ( $\mathrm{HR}=1.90,95 \% \mathrm{Cl} 1.32$ to 2.72 ) or prefrail ( $\mathrm{HR}=1.56,95 \% \mathrm{Cl} 1.10$ to 2.20) was associated with increased mortality. There was some evidence that the HR for polypharmacy on mortality was lower among frailer individuals, but the overall polypharmacy by frailty interaction was not statistically significant $(p=0.102)$. Conclusions For those with cognitive impairment, greater concern should be afforded to the number of medications than the prescription of specific classes. Frailer individuals may have a lower relative risk of mortality associated with polypharmacy than less frail individuals.

\section{INTRODUCTION}

More than 850000 people live with dementia in the UK and many more have significant
Strengths and limitations of this study

- This study followed a large population-representative cohort of people with cognitive impairment for up to 8 years.

- All medication use was included, both over-thecounter and prescribed.

- Demographic-related and health-related confounding variables were included in the analysis.

- There was no loss to follow-up as each participant was flagged for notification of mortality with the Office for National Statistics.

- Medication use and frailty were only ascertained once at baseline, ascertainment of medications relied on self-report with interviewers checking packaging and prescriptions where available.

cognitive impairment. ${ }^{1}$ Around $70 \%$ of people with dementia live with comorbidity and are prescribed multiple medications. ${ }^{2}$ Polypharmacy, often defined as the regular use of five or more medications, is common among older adults and people with dementia, particularly as clinical prescribing guidelines often take a single disease focus, rarely accounting for patients with multimorbidity. ${ }^{3}$

Polypharmacy has been associated with incident frailty. ${ }^{4}$ Frailty is often used to describe low physiological reserve and individual susceptibility to stressors that may affect health ${ }^{56}$ and is an important determinant of health outcomes in older people, including hospitalisation and mortality. ${ }^{7-9}$ Incorporating frailty assessment into primary care is increasingly encouraged to identify individuals at greater risk of mortality, hospitalisation and susceptibility to adverse health outcomes. ${ }^{10}$ An important aspect of the validity of frailty measures is whether they can predict resilience or susceptibility to other potential risk factors. ${ }^{9}$ Recent findings 
suggest that polypharmacy and frailty are associated with mortality, incident disability and hospitalisation compared with non-frail participants without polypharmacy. ${ }^{11}$ However, the moderating role of frailty in the relationship between polypharmacy and adverse outcomes, hence whether 'frailty' can be used to identify those at particular risk from polypharmacy, is unknown.

All medications have associated risks; a medication is considered a 'potentially inappropriate medication' (PIM) when the potential harm outweighs potential benefit among a particular group. PIMs are associated with increased risk of hospitalisation, mortality and other adverse health outcomes ${ }^{12-14}$ and around $29 \%$ of older people and $64 \%$ of people with dementia are prescribed at least one PIM. ${ }^{15}{ }^{16}$ Validated criteria can be used to identify potentially inappropriate prescribing, examples include the Beers criteria, which is primarily used in the USA, and the European Screening Tool for Older Persons Prescribing (STOPP). ${ }^{17} 18$ These criteria identify PIM for specific patient groups, where interaction with specific diseases, prescriptions or overuse may result in adverse effects.

Proton pump inhibitors (PPIs) are considered potentially inappropriate after 8 weeks in older adults according to STOPP criteria. PPIs are prescribed extensively and although generally considered safe and effective, recent evidence has linked PPIs with potential adverse events, including hip and spine fractures, community-acquired pneumonia, Clostridium difficile infections, and vitamin and mineral deficiencies. ${ }^{19-26}$ PPIs are considerable contributors to polypharmacy, with $27 \%$ of patients using PPIs being prescribed them long-term, despite longterm effects being unknown. ${ }^{27}$ Understanding the longterm effect of PPI use may improve patient safety, reduce unnecessary polypharmacy and have significant financial implications for health services.

The STOPP criteria also identify groups of PIM with central nervous system effects, and PIMs that are particularly inappropriate for people with dementia. People with dementia may be exposed to central nervous system acting medications, including antipsychotics, antidepressants, benzodiazepines (BZDs) and medications with anticholinergic effects in order to treat comorbidities and manage behavioural and psychological symptoms. However, these medications are associated with adverse cardiovascular effects, ${ }^{28}$ hypotension, falls ${ }^{29-31}$ confusion, detrimental effects on cognition ${ }^{32}$ and mortality, ${ }^{33}$ and are considered potentially inappropriate in both STOPP and Beers criteria. People with dementia may be more susceptible to adverse effects of central nervous system acting medications due to age-related and disease-related pharmacokinetic and pharmacodynamic changes, including alterations in the blood-brain barrier permeability associated with Alzheimer's disease. ${ }^{34-36}$

Being frail may also increase susceptibility to adverse effects of PIM and polypharmacy, since frailty is defined as increased vulnerability to risk factors and lack of physiological reserve. ${ }^{5}$ Using frailty criteria may, therefore, enable the identification of those at particular risk from PIM and polypharmacy, although whether frailty exacerbates any adverse effects of PIM is not known.

This study seeks to understand the effect of potentially inappropriate use of central nervous system acting drugs, PPIs and polypharmacy among cognitively impaired older adults, and whether any risk varies with increasing frailty. We used the Cognitive Function and Ageing Study II (CFAS II) to estimate (a) the association between PIMs and polypharmacy at baseline and survival up-to 8 years, and (b) the moderating role of frailty in this relationship.

\section{METHODS}

CFAS II is a cohort study of ageing in England. The design and methods have been described in detail elsewhere. ${ }^{37} \mathrm{In}$ short, participants were randomly sampled from primary care lists within three geographic centres representing urban and rural areas (Nottingham, Cambridgeshire and Newcastle) between 2008 and 2011. In England, the vast majority of people are registered with a single primary care provider, and so primary care patient lists form sampling frames that are close to enumerations of the populations of specific areas. Approximately, 2500 participants were recruited from each geographic area. All participants were aged 65 years or older at baseline and were interviewed in their usual place of residence, whether this was their own home or a long-term care facility, by a trained interviewer. The CFAS interview included questions on health and lifestyle, demographics, and current medication use, as well as a range of cognitive tests and algorithmic dementia assessment. This was repeated at a two-year follow-up assessment.

\section{Sample}

For the present study, we used data from all participants with a baseline Mini-Mental State Examination (MMSE) score of 24 or lower, indicating clinically significant cognitive impairment. ${ }^{38}$ Participants were excluded if medication data were not recorded or were deemed unreliable, that is, if MMSE was less than 18 and proxy-reported medication data were unavailable.

\section{Exposures}

\section{Medication use}

Information on medication use was ascertained from the question: 'Do you take any medicine, tablets or injections of any kind, that either you buy yourself or that are prescribed by your doctor?' To improve data accuracy, reported medications were cross-checked with medication packs by the interviewer. In care homes, staff crosschecked with medication lists. Medications were coded using Read Codes and Application Dictionary. ${ }^{39}$

\section{Potentially inappropriate medications}

PIMs were identified using STOPP criteria. ${ }^{18}$ We focused primarily on medications from Section D: Central Nervous System Criteria of STOPP, including antidepressants, 
antipsychotics, other anticholinergics (ie, anticholinergics other than antidepressants and antipsychotics) and BZDs. Acetylcholinesterase inhibitors, inappropriate in patients with vascular dementia, were not included in this study due to specific dementia subtype diagnosis information being unavailable. PPIs, indicated as potentially inappropriate in long-term use, contributors to polypharmacy and a priority of deprescribing, were also included. ${ }^{19} 40$

Anticholinergic medications were defined using the anticholinergic cognitive burden scale (ACB). Medications with definite anticholinergic activity, classified as an ACB score of 3, were included. ${ }^{41}$ PIMs were then grouped into mutually exclusive categories of: (a) antipsychotic, (b) anticholinergic (excluding antipsychotics and antidepressants), (c) tricyclic antidepressants, (d) other antidepressants, (e) BZDs and (f) PPI (see online supplementary appendix 1 containing medication code lists).

\section{Polypharmacy}

Total number of prescribed and over-the-counter medications was calculated from medication lists collated during interviews. This number excluded the specific PIM described above, to allow for mutually exclusive exposures comparing PIM to polypharmacy and to understand the effect of polypharmacy on mortality, independent of specific PIM use. Polypharmacy was categorised as the use of between five and nine such medications, hyperpolypharmacy as more than ten in line with previous studies. ${ }^{1142}$

\section{Time-varying exposure to PIM}

To test the extent to which the exposure varied with time, the use of PIM at baseline and 2-year follow-up was compared, among the subsample for whom medication data were available at the 2-year follow-up interview.

\section{Frailty}

The frailty phenotype as first described by Fried $e t a \tilde{p}$ was used to measure frailty. The frailty phenotype is known to predict adverse outcomes in a general older population. ${ }^{43}$ This definition of frailty incorporates measures from multiple domains reflecting physiological decline and consists of five components: slowness, weakness, exhaustion, weight loss and low physical activity. Individuals are deemed 'frail' if they are positive on three, four or five components, 'prefrail' if positive on one or two and 'not frail' otherwise. We could not operationalise frailty exactly as originally performed in the Cardiovascular Health Study, ${ }^{5}$ but in common with other epidemiological studies of ageing ${ }^{44} 45$ each component was operationalised as closely as possible using available data, as follows:

Slowness was measured using the average walking speed from two gait speed tests across 2.4 metres. Participants falling within the slowest quartile of the whole CFAS II sample were considered impaired for this component. Participants who did not consent or could not complete the test were also considered impaired.
Weakness was assessed using the timed sit-to-stand test. ${ }^{46}$ Participants were asked to stand from sitting five times, without using their arms. Participants who did not consent, could not complete the test and those within the slowest quartile of time among completers were considered impaired.

Exhaustion was self-reported in answer to the question 'Do you get worn out/exhausted towards the evening?' If there was no response, interviewer rating of observed slow movements not due to physical illness was used. Both questions were measured with three response categories (no/mild/severe). Participants with mild or severe exhaustion, not explained by strenuous activities, were coded as frail on this criterion.

Weight loss was assessed by self-report. Participants reporting losing $10 \mathrm{lbs}(4.5 \mathrm{~kg})$ or more in $\leq 6$ months prior to the interview were classified as positive on this criterion.

Low physical activity was assessed through self-reported time spent doing vigorous, moderate or mildly energetic activities. This was scored based on responses to a series of questions about time spent in specific activities, including, for example, swimming, jogging, walking, gardening and housework (see online supplementary appendix 2 for a full list). A score of 1 was allocated for each activity if a participant reported doing an activity hardly ever or never, less than three times a month or not engaging in the activity at all, and 0 if more frequently. The scores across activities were summed to create a continuous variable, where higher scores indicated less activity. Participants in the least active quartile were coded positive on this criterion.

\section{Other covariates}

Multivariate analyses were adjusted for age, sex, living situation (care home, residential homes, nursing homes and long-stay hospitals, or own home), cognitive impairment (MMSE score) and number of self-reported comorbidities (angina, arthritis, asthma, cancer, chronic bronchitis, depression, diabetes, epilepsy, heart attack, high blood pressure, intermittent claudication, low blood pressure, Parkinson's disease, peptic ulcers, pernicious anaemia and stroke).

\section{Outcomes}

CFAS II participants were flagged on the UK Office for National Statistics National Health Service Central Register for notification of date of death, and so survival status is known for all participants. The current analysis includes all deaths up to 31 October 2016, providing up to 8 years follow-up.

\section{Statistical analysis \\ Univariate analysis}

Kaplan-Meier curves estimated survival probabilities from baseline until censoring or death. Logrank tests were used to test survival differences between users and non-users of each PIM. Participants reporting polypharmacy and 
hyperpolypharmacy were compared with those reporting zero to four medications.

Cox regression models estimated univariate HRs $(95 \%$ CIs) for each exposure. Participants entered the analysis at the time of baseline interview, and were followed until death or censoring on 31st October 2016. Time since the interview was the time scale for all analyses.

\section{Multivariate analysis}

Two Cox proportional hazards regression models estimated independent effects of each PIM and polypharmacy, controlling for covariates. Model 1 adjusted for age, sex, cognitive impairment, living situation and comorbidities. Model 2 included a categorical frailty variable (non-frail, prefrail and frail). Sensitivity analyses included adding individual frailty components in Cox multivariate regressions to assess their association with mortality, and adjusting for each comorbid condition individually rather than as a single cumulative variable. The proportional hazards assumption was tested by inspection of Schoenfeld residuals. ${ }^{47}$

\section{Frailty as a moderator}

First, multivariate analyses as described above were stratified by baseline frailty status. Interaction terms were then added to the full model to test the moderating effect of frailty on the link between each PIM, polypharmacy and mortality. Likelihood-ratio tests assessed the statistical significance of PIM by frailty and polypharmacy by frailty interactions.

The data set analysed during the current study was provided by the CFAS. Details of how to request CFAS data are available (http://www.cfas.ac.uk/cfas-ii/cfasiidata/). All analyses were performed using Stata V.14.

\section{RESULTS}

Of the 7762 CFAS II participants, 1154 met inclusion criteria (see figure 1). Within the final sample, $62 \%$ were female, mean age was 79 years $(\mathrm{SD}=7.4)$ and 154 $(13 \%)$ had a study diagnosis of dementia (see table 1). Participants reported a mean average of $2.4 \quad(\mathrm{SD}=1.6)$ comorbidities, $5.5(\mathrm{SD}=3.6)$ regular medications and $44 \%$ reported taking at least one PIM. PPIs were most frequent $(n=331,28.7 \%)$, followed by 'other antidepressants' ( $\mathrm{n}=119,10.3 \%)$, 'tricyclic antidepressants' $(\mathrm{n}=76$, $6.6 \%)$, anticholinergics $(\mathrm{n}=71,6.2 \%)$, BZDs $(\mathrm{n}=46,4.0 \%)$ and antipsychotics $(\mathrm{n}=21,1.8 \%)$. There were $493(42.7 \%)$ participants taking 5-9 medications (polypharmacy) and $110(9.5 \%)$ taking $\geq 10$ medications, excluding PIMs.

\section{Survival analyses}

\section{Univariate analyses}

Up to 31 October 2016, 489 (42.4\%) participants had died; the median follow-up time was 5.6 years. Among participants reporting PIM, 73\% ( $\mathrm{n}=11)$ of antipsychotic users, $52 \%(\mathrm{n}=37)$ of users of other anticholinergics, $43 \%$ $(\mathrm{n}=33)$ of users of tricyclic antidepressants, $46 \% \quad(\mathrm{n}=21)$

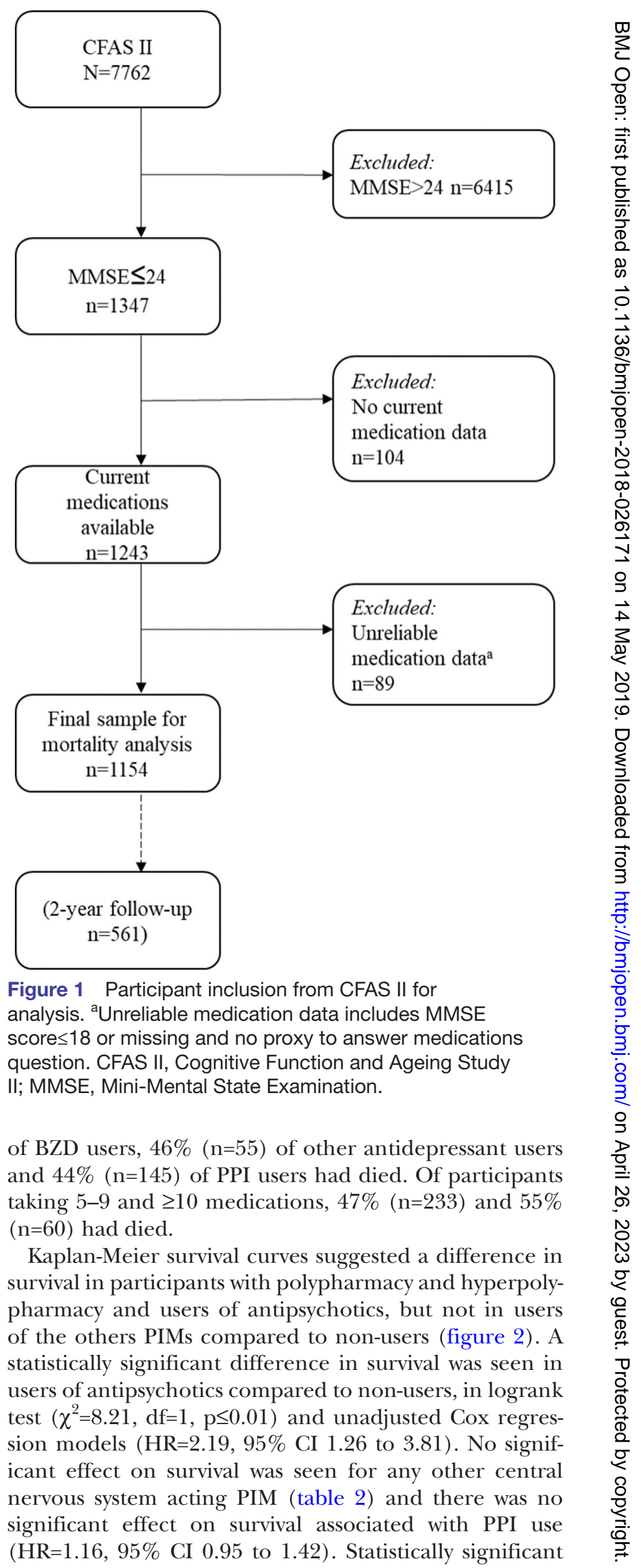

Porter B, et al. BMJ Open 2019;9:e026171. doi:10.1136/bmjopen-2018-026171 
Table 1 Sample characteristics by frailty classification. Cognitive Function and Ageing Study II

\begin{tabular}{|c|c|c|c|c|}
\hline \multirow[b]{2}{*}{ Characteristics } & \multirow[b]{2}{*}{$\begin{array}{l}\text { Total sample } \\
\mathrm{n}=1154\end{array}$} & \multicolumn{3}{|c|}{ Characteristics within frailty classifications } \\
\hline & & $\begin{array}{l}\text { Non-frail } \\
n=204\end{array}$ & $\begin{array}{l}\text { Prefrail } \\
n=530\end{array}$ & $\begin{array}{l}\text { Frail } \\
n=420\end{array}$ \\
\hline Gender (\% female) & $717(62.1)$ & $96(47.1)$ & $314(59.3)$ & $307(73.1)$ \\
\hline Mean age (years, SD) & $78.8(7.4)$ & $75.7(6.5)$ & $78.8(7.4)$ & $80.4(7.3)$ \\
\hline \multicolumn{5}{|l|}{ PIMs (count, \%) } \\
\hline Antipsychotics & $21(1.8)$ & $4(2.0)$ & $7(1.3)$ & $10(2.4)$ \\
\hline Anticholinergics & $78(6.8)$ & $6(2.9)$ & $36(6.8)$ & $36(8.6)$ \\
\hline Tricyclic antidepressants & $76(6.6)$ & $5(2.5)$ & $30(5.6)$ & $41(9.8)$ \\
\hline Other antidepressants & $119(10.3)$ & $14(6.9)$ & $35(6.6)$ & $70(16.7)$ \\
\hline Benzodiazepines & $46(4.0)$ & $4(2.0)$ & $14(2.6)$ & $28(6.7)$ \\
\hline Proton pump inhibitors & $331(28.7)$ & $34(16.7)$ & $149(28.1)$ & $148(35.2)$ \\
\hline \multicolumn{5}{|l|}{ Polypharmacy } \\
\hline $0-4$ & $551(47.8)$ & $143(70.1)$ & $260(49.1)$ & $148(35.2)$ \\
\hline $5-9$ & $493(42.7)$ & $56(27.5)$ & $229(43.2)$ & $208(49.5)$ \\
\hline $10+$ & $110(9.5)$ & $5(2.5)$ & $41(7.7)$ & $64(15.2)$ \\
\hline \multicolumn{5}{|l|}{ Cognitive impairment } \\
\hline Dementia (count, \%) & $154(13.3)$ & $16(7.8)$ & $79(14.9)$ & $59(14.1)$ \\
\hline \multicolumn{5}{|l|}{ MMSE score (count, \%) } \\
\hline $22-24$ & $789(68.4)$ & $164(80.4)$ & $362(68.3)$ & $263(62.6)$ \\
\hline $21-19$ & $283(24.5)$ & $37(18.1)$ & $127(24.0)$ & $119(28.3)$ \\
\hline$\leq 18$ & $82(7.1)$ & $3(1.5)$ & $41(7.7)$ & $38(9.1)$ \\
\hline Number of comorbidities (mean, SD) & $2.4(1.6)$ & $1.6(1.2)$ & $2.3(1.5)$ & $2.9(1.7)$ \\
\hline Care home resident (count, \%) & $47(4.1)$ & $2(1.0)$ & $22(4.2)$ & $23(5.5)$ \\
\hline \multicolumn{5}{|l|}{ Frailty individual components (count, \%) } \\
\hline Low physical activity & $313(27.1)$ & - & $58(10.9)$ & $255(60.7)$ \\
\hline Weight loss & $122(10.6)$ & - & $29(5.5)$ & $93(22.1)$ \\
\hline Weakness* & $802(69.5)$ & - & $388(73.2)$ & $414(98.6)$ \\
\hline Slowness† & $702(60.8)$ & - & $313(59.1)$ & 389 (92.6) \\
\hline Exhaustion & 334 (28.9) & - & 74 (14.0) & 260 (61.9) \\
\hline Death before 31 October 2016 (count, \%) & 489 (42.4) & $45(22.1)$ & $226(42.6)$ & $218(51.9)$ \\
\hline
\end{tabular}

*Missing $n=603$ : categorised as frail if missing due to inability to complete the test.

†Missing $n=244$ : categorised as frail if missing due to inability to complete the test.

MMSE, Mini-Mental State Examination score; PIMs, potentially inappropriate medications.

difference in survival was seen across polypharmacy categories $\left(\chi^{2}=23.15, \mathrm{df}=2, \mathrm{p} \leq 0.01\right)$. Polypharmacy and hyperpolypharmacy were both significantly associated with mortality, HR=1.39 (95\% CI 1.14 to 1.70$)$ and $\mathrm{HR}=1.95$ (95\% CI 1.45 to 2.61 ), respectively (see table 2 ).

Multivariable analyses

The association between antipsychotics and mortality remained after adjusting for demographic variables, comorbidities and cognitive impairment (HR=3.24, 95\% CI 1.83 to 5.73) and after adjusting for frailty. After adjusting for confounders, no significant association with mortality was evident in the other central nervous system acting PIM or PPIs (see table 2). Adjustment for confounders explained about half of the univariable effect of polypharmacy such that it was no longer statistically significant (multivariate model $\mathrm{HR}=1.21,95 \% \mathrm{CI}$ 0.97 to 1.50 ); however, hyperpolypharmacy remained significantly associated with mortality in both adjusted models (frailty model: $\mathrm{HR}=1.60,95 \%$ CI 1.16 to 2.22 ) (see table 2). The proportional hazards assumption, assessed using Schoenfeld residuals, was not violated in this mode $1\left(\chi^{2}=16.91, \mathrm{df}=15, \mathrm{p}=0.324\right)$. There was no substantial change in estimates when comorbid conditions were included individually in the main multivariate model (see online supplementary file appendix 3).

\section{Continuation of medication use}

At the 2-year follow-up, 561 (48.6\%) had survived and provided medication data. Among these, 69\% ( $\mathrm{n}=29)$ of 

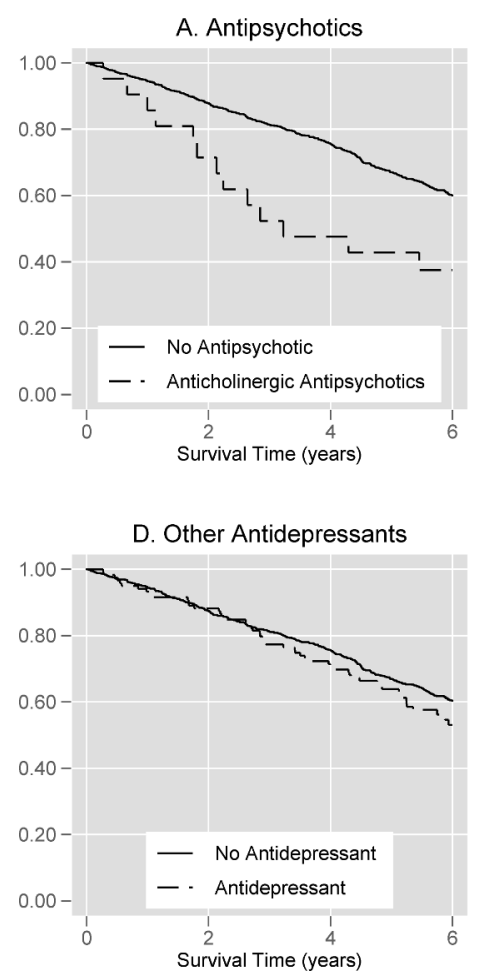
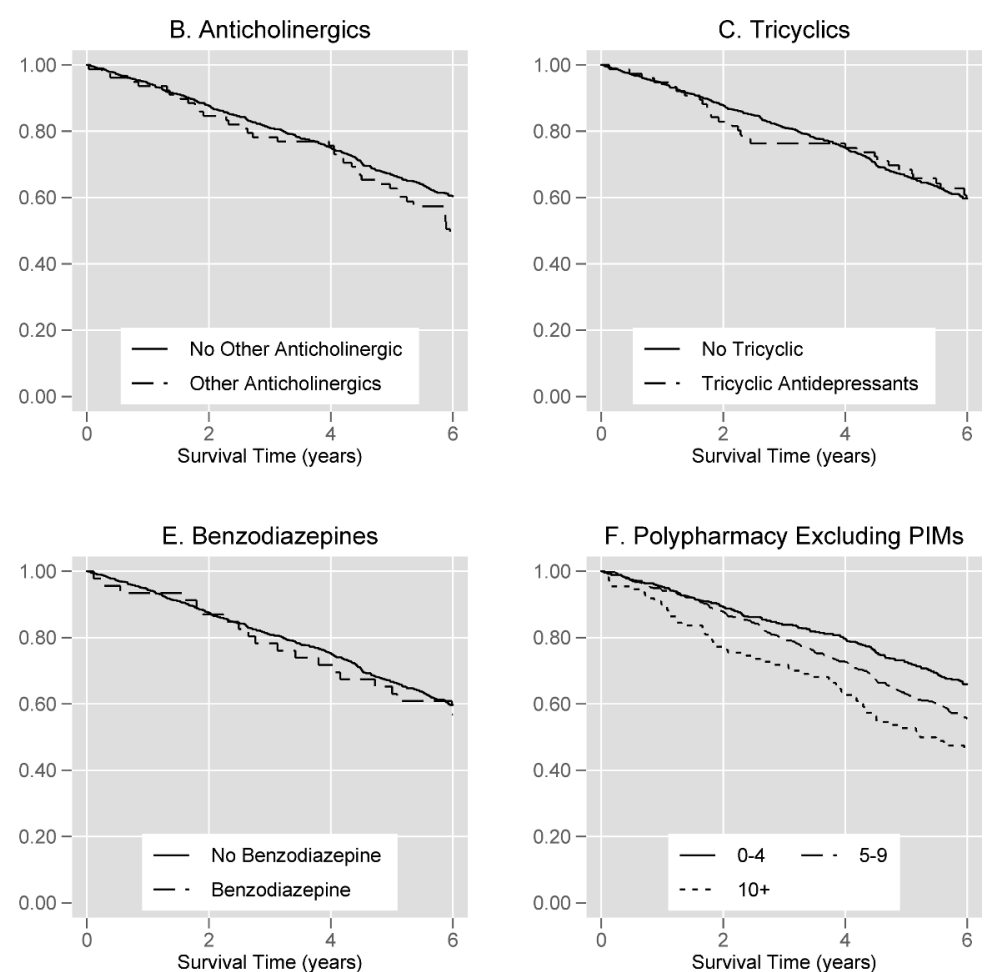

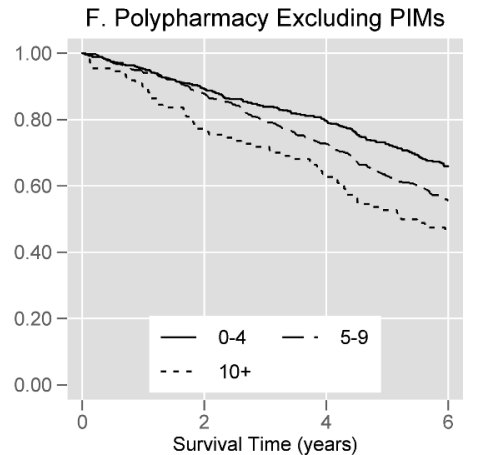

Figure 2 Kaplan-Meir survival curves showing the proportion of patients surviving with time, stratified by baseline use of PIM. (A) Shows the effect of antipsychotics, (B) the effect of anticholinergics apart from antipsychotics and antidepressants, (C) the effect of tricyclics, (D) other antidepressants, (E) benzodiazepines and (F) the total number of other drugs coded as polypharmacy (5-9 drugs) or hyperpolypharmacy (10 or more) excluding any of the categories previously described. PIM, potentially inappropriate medication.

those reporting anticholinergics at baseline also reported anticholinergics at 2 years, $60 \%(\mathrm{n}=3)$ continued antipsychotics, $59 \%(\mathrm{n}=13)$ continued BZDs, $77 \% \quad(\mathrm{n}=31)$ continued tricyclics and $85 \%(n=136)$ of those using PPI at baseline were also using PPI at follow-up. This suggests that the majority of medication use reported at baseline reflected relatively long-term use.

\section{Frailty}

At baseline, $36.4 \%$ of the sample were classified as frail $(n=420)$, while $45.9 \%$ were prefrail $(n=530)$. PIM use was less frequently reported among the non-frail $(17.7 \%)$ group compared with prefrail $(27.7 \%)$ and frail $(32.1 \%)$ participants. At the point of censoring, $22.1 \%(\mathrm{n}=45)$ of non-frail, $42.6 \%(\mathrm{n}=226)$ prefrail and $51.9 \%(\mathrm{n}=218)$ of frail participants had died.

In univariate analyses, prefrail or frail participants were at a significantly increased risk of mortality compared with non-frail (prefrail: $\mathrm{HR}=2.22,95 \%$ CI 1.59 to 3.09; frail: $\mathrm{HR}=2.92,95 \%$ CI 2.09 to 4.07 ). Being frail and prefrail remained significantly associated with mortality in the fully adjusted model (frail: HR=1.90, 95\% CI 1.32 to 2.71; prefrail: $\mathrm{HR}=1.56,95 \%$ CI 1.11 to 2.20 ) (see table 2).

\section{Frailty as moderator of the effect of PIM and polypharmacy}

Stratifying multivariate models by frailty status suggested similar relative risk of mortality among antipsychotic users at all levels of frailty. The HR associated with antipsychotic use among non-frail users was 3.60 (95\% CI 0.40 to 31.99$)$, was 2.89 (95\% CI 1.26 to 6.66 ) in prefrail and was 3.34 (95\% CI 1.37 to 8.12$)$ in frail antipsychotic users (table 3).

Frailty and polypharmacy interaction terms suggested worse mortality associated with polypharmacy and hyperpolypharmacy in people who were not frail compared with those who were prefrail or frail (table 3). However, this interaction was not statistically significant (likelihood-ratio test for interaction: $\left.\chi^{2}=7.73, \mathrm{df}=4, \mathrm{p}=0.102\right)$.

The association between BZDs and mortality significantly varied across frailty groups, (likelihood-ratio test for interaction: $\left.\chi^{2}=6.05, \mathrm{df}=2, \mathrm{p}=0.049\right)$ and suggested an inverse relationship between BZD use and mortality among the frail $(\mathrm{HR}=0.43)$, but no effect among the prefrail or not frail participants (table 3).

\section{Estimating the effect of individual frailty components}

Including frailty components individually in adjusted analysis instead of the composite measure made little difference to HRs estimated from previous models. As individual markers of frailty, low physical activity, weakness (measured by chair-rise test) and slowness (measured by the gaitspeed test) were individually associated with mortality (low physical activity: $\mathrm{HR}=1.41,95 \%$ CI 1.15 to 1.71 ; weakness: $\mathrm{HR}=2.23,95 \%$ CI 1.77 to 2.83 and slowness: $\mathrm{HR}=1.99,95 \%$ CI 1.62 to 2.45$)$. However, slow gait was the only component independently associated with mortality in the multivariate model (HR=1.42, 95\% CI 1.12 to 1.81$)$ (table 4$)$. 
Table 2 Unadjusted and adjusted potentially inappropriate medication (PIM) use HRs for survival, univariate and multivariate models. Cognitive Function and Ageing Study II $n=1154$

\begin{tabular}{|c|c|c|c|}
\hline Variables & Univariate & Multivariate & Frailty model† \\
\hline Antipsychotics & $2.19^{*}(1.26$ to 3.81$)$ & $3.24^{\star}(1.83$ to 5.73$)$ & $3.28^{*}(1.85$ to 5.80$)$ \\
\hline Anticholinergics & 1.29 (0.92 to 1.80$)$ & 1.18 (0.83 to 1.67$)$ & $1.17(0.83$ to 1.66$)$ \\
\hline Other antidepressants & 1.21 (0.91 to 1.61$)$ & 0.94 (0.70 to 1.28$)$ & $0.90(0.66$ to 1.22$)$ \\
\hline Proton pump inhibitor & 1.16 (0.95 to 1.42$)$ & 1.08 (0.88 to 1.33$)$ & $1.05(0.86$ to 1.30$)$ \\
\hline \multicolumn{4}{|l|}{ Polypharmacy } \\
\hline $0-4$ & 1 & 1 & 1 \\
\hline $5-9$ & $1.39 *(1.14$ to 1.70$)$ & 1.21 (0.97 to 1.50$)$ & $1.17(0.95$ to 1.45$)$ \\
\hline Gender (female) & $0.76^{\star}(0.63$ to 0.91$)$ & $0.62^{*}(0.51$ to 0.75$)$ & $0.57^{*}(0.46$ to 0.69$)$ \\
\hline MMSE score (per point) & $0.88^{*}(0.85$ to 0.90$)$ & $0.91^{*}(0.88$ to 0.94$)$ & $0.91^{*}(0.89$ to 0.94$)$ \\
\hline Care home residence & $2.36^{*}(1.63$ to 3.42$)$ & 1.23 (0.83 to 1.84$)$ & $1.20(0.81$ to 1.80$)$ \\
\hline Comorbidities & $1.06^{*}(1.00$ to 1.13$)$ & $1.08^{*}(1.01$ to 1.15$)$ & 1.05 (0.98 to 1.13$)$ \\
\hline \multicolumn{4}{|l|}{ Frailty } \\
\hline Prefrail & $2.22^{*}$ (1.59 to 3.09$)$ & & $1.56^{*}$ (1.11 to 2.20$)$ \\
\hline Frail & $2.92^{*}(2.09$ to 4.07$)$ & & $1.90^{*}(1.32$ to 2.72$)$ \\
\hline
\end{tabular}

$\mathrm{HR}(95 \% \mathrm{Cl})$.

${ }^{*} \mathrm{P}<0.05$.

†Multivariate model adjusted for frailty.

MMSE, Mini-Mental State Examination score (0-30 with higher scores indicating better cognition).

\section{DISCUSSION}

This study estimated the association between PIMs, polypharmacy and risk of mortality among cognitively impaired older adults and the moderating role of frailty in this relationship. We found that, as expected, use of antipsychotic medications, polypharmacy and hyperpolypharmacy were associated with an increased risk of mortality, but no other specific PIM we tested was linked to the risk of death. The excess risk associated with hyperpolypharmacy and with antipsychotics remained after adjustment for frailty status. Polypharmacy was more common among frailer participants. Contrary to expectation, the HR for death associated with polypharmacy appeared higher among the non-frail population than among the prefrail or frail, with excess risk of death associated with using five to nine medications seeming almost exclusively restricted to the least physically frail. The frailty phenotype did independently predict mortality, but when analysed individually slow gait speed was the only component of the frailty measure that independently predicted mortality in this cohort.

The link between antipsychotic medication and mortality in people with dementia is well known ${ }^{48}$ and our result extends this finding to a broader group with cognitive impairment. Previous studies have also found an increased risk of death associated with increased anticholinergic burden. ${ }^{33}$ Since anticholinergic medications encompasses a wide variety of medications, including many antidepressants and antipsychotics, in this analysis, anticholinergics were subclassified into mutually exclusive categories to understand the contribution of specific medication classes. Our finding that only antipsychotic medications were associated with increased mortality, compared with tricyclic antidepressants or other anticholinergic medications, indicates a potential class-specific effect, such that antipsychotic use may be the driver of previously observed associations between anticholinergics and mortality when anticholinergics are considered together. ${ }^{49}$ Hence, as previously suggested elsewhere, ${ }^{50}$ considering 'anticholinergics' as a class with respect to adverse outcomes might not be meaningful.

We found no evidence for an association between PPI use and mortality in this cognitively impaired population. PPIs are highly prevalent and are often overused without appropriate indication. ${ }^{275152}$ For people with comorbidities and polypharmacy, who are taking PPIs on a longterm and potentially inappropriate basis, there may also be a risk of drug-drug interactions, which can lead to 
Table 3 HRs estimated by Cox regression models for association between potentially inappropriate medication (PIM) use and mortality, adjusted for age, gender, MMSE score, care home residence and comorbidities and stratified by frailty. Data from the Cognitive Function and Ageing Study II

\begin{tabular}{|c|c|c|c|c|}
\hline \multirow[b]{2}{*}{ Variables } & \multicolumn{3}{|c|}{ Cox regression analysis stratified by frailty classification $\dagger$} & \multirow[b]{2}{*}{$\begin{array}{l}\text { Likelihood ratio } \\
\chi^{2} \text { ( } \text { value) }\end{array}$} \\
\hline & $\begin{array}{l}\text { Not frail } \\
n=204\end{array}$ & $\begin{array}{l}\text { Prefrail } \\
n=530\end{array}$ & $\begin{array}{l}\text { Frail } \\
n=420\end{array}$ & \\
\hline \multicolumn{5}{|l|}{ PIM } \\
\hline Antipsychotic & $3.60(0.40$ to 31.99$)$ & $2.89^{*}(1.26$ to 6.66$)$ & $3.34^{*}(1.37$ to 8.12$)$ & $0.01(0.995)$ \\
\hline Tricyclic antidepressant & 0 & $1.84(0.98$ to 3.44$)$ & $0.90(0.55$ to 1.48$)$ & $5.63(0.060)$ \\
\hline Anticholinergics & $1.29(0.16$ to 10.61$)$ & $1.05(0.61$ to 1.79$)$ & $1.23(0.76$ to 2.01$)$ & $0.32(0.854)$ \\
\hline Other antidepressants & $0.86(0.23$ to 3.20$)$ & 1.12 (0.67 to 1.89$)$ & 0.74 (0.49 to 1.12$)$ & $2.93(0.230)$ \\
\hline Benzodiazepines & $0.92(0.11$ to 7.78$)$ & $1.40(0.66$ to 2.97$)$ & $0.43^{*}(0.21$ to 0.86$)$ & $6.05(0.049)^{\star}$ \\
\hline Proton pump inhibitor & 1.05 (0.43 to 2.59$)$ & 1.04 (0.76 to 1.42$)$ & 1.09 (0.80 to 1.49$)$ & $0.43(0.808)$ \\
\hline \multicolumn{5}{|l|}{ Polypharmacy } \\
\hline $0-4$ & 1 & 1 & 1 & 7.73 \\
\hline $5-9$ & $2.34^{*}(1.16$ to 4.70$)$ & $1.07(0.78$ to 1.45$)$ & $1.17(0.84$ to 1.64$)$ & $(0.102)$ \\
\hline $10+$ & 4.22 (0.90 to 19.90$)$ & 1.31 (0.75 to 2.29$)$ & $1.80^{*}$ (1.15 to 2.82$)$ & \\
\hline \multicolumn{5}{|l|}{ Covariates } \\
\hline Age at interview & $1.13^{*}(1.07$ to 1.19$)$ & $1.11^{*}(1.09$ to 1.13$)$ & $1.08^{*}(1.06$ to 1.11$)$ & \\
\hline Gender (female) & 0.71 (0.35 to 1.43$)$ & $0.67^{\star}(0.50$ to 0.89$)$ & $0.43^{*}(0.31$ to 0.58$)$ & \\
\hline MMSE score (per point) & $0.87^{*}(0.77$ to 0.98$)$ & $0.92^{*}$ (0.88 to 0.96$)$ & $0.91^{*}(0.87$ to 0.96$)$ & \\
\hline Care home residence & 0.00 & $0.92(0.50$ to 1.71$)$ & 1.68 (0.98 to 2.88$)$ & \\
\hline Comorbidities & $1.16(0.90$ to 1.50$)$ & 1.03 (0.93 to 1.14$)$ & 1.02 (0.92 to 1.12$)$ & \\
\hline
\end{tabular}

$\operatorname{HR}(95 \% \mathrm{Cl})$.

${ }^{*} \mathrm{P}<0.05$.

†Classified as positive on individual frailty components if score, rating or time within the upper quartile. Classified as frail if positive on 3-5 individual frailty components and prefrail if 1-2 components.

łLikelihood ratio test comparing exposure-frailty interaction models with multivariate frailty model with prefrail group as baseline.

MMSE, Mini-Mental State Examination score (0-30 with higher scores indicating better cognition).

a range of adverse effects. Deprescribing inappropriate PPIs among older people is considered a priority ${ }^{40}$ and while we did not find PPI use was associated with survival, there is building evidence suggesting PPIs are associated with other clinically important adverse effects including C. difficile and increased risk of fractures. ${ }^{27} 53$

In this novel investigation of the role of frailty on the effect of PIM in older adults with cognitive impairment, we found some evidence of a moderating role in that the HR for polypharmacy was higher among the least physically frail individuals. This may be partly caused by a lower risk of death from other causes among the less frail groups, so that if polypharmacy caused a similar increase in absolute risk of mortality across groups, this would correspond to a lower relative risk among the frailer participants.

BZD use was associated with better survival in frail participants. Long-term use of BZDs is discouraged; however, more than two-thirds of our participants using BZD at baseline were still using BZD 2 years later, suggesting that much of the BZD use recorded in our study is long-term. It is possible that prescribing behaviours around BZDs may differ compared with other PIMs. This could include improved monitoring of efficacy and safety leading to subsequent apparent protective effects. Alternatively, this finding might represent residual confounding such that BZDs are prescribed to those with a lower risk of death, an effect of BZDs causing otherwise healthy people to be classified as frail, or it is possible that this is a chance association given the large numbers of individual hypotheses being tested and borderline statistical significance of the interaction effect. In any case, there is no evidence from this study that BZDs or other individual PIM apart from antipsychotics are associated with excess mortality among people with cognitive impairment.

Previous studies have described that polypharmacy and hyperpolypharmacy are associated with frailty ${ }^{4}$ and that frailty and polypharmacy are associated with mortality, incident disability and hospitalisation. ${ }^{1142}$ While including frailty and polypharmacy interactions in our analysis did indicate a significantly increased risk of mortality in frail, hyperpolypharmacy participants, the greatest risk was observed in non-frail participants with polypharmacy. This interaction effect was not statistically significant in our study; however, a cohort study of men aged 70 years and older characterised transitions across frailty states and death and similarly found that additional medications 
Table 4 Risk of mortality associated with the use of PIM and individual frailty components in univariate and multivariate models. Cognitive Function and Ageing Study II $n=1154$

\begin{tabular}{|c|c|c|}
\hline Variables & $\begin{array}{l}\text { Univariate } \\
\text { HR }(95 \% \mathrm{Cl})\end{array}$ & $\begin{array}{l}\text { Multivariate† } \\
\text { HR }(95 \% \mathrm{Cl})\end{array}$ \\
\hline Antipsychotic & $2.19^{*}(1.26$ to 3.81$)$ & $3.30^{*}(1.86$ to 5.86$)$ \\
\hline $\begin{array}{l}\text { Tricyclic } \\
\text { antidepressant }\end{array}$ & 1.01 (0.70 to 1.47$)$ & $1.04(0.71$ to 1.53$)$ \\
\hline Anticholinergic & 1.29 (0.92 to 1.80$)$ & 1.17 (0.83 to 1.66$)$ \\
\hline $\begin{array}{l}\text { Other } \\
\text { antidepressants }\end{array}$ & 1.21 (0.91 to 1.61$)$ & 0.88 (0.65 to 1.20$)$ \\
\hline Benzodiazepines & 1.08 (0.67 to 1.72$)$ & 0.73 (0.46 to 1.18$)$ \\
\hline PPI & $1.16(0.95$ to 1.42$)$ & 1.05 (0.85 to 1.29$)$ \\
\hline \multicolumn{3}{|l|}{ Polypharmacy } \\
\hline $0-4$ & 1 & 1 \\
\hline $5-9$ & $1.39^{*}(1.14$ to 1.70$)$ & 1.17 (0.95 to 1.46$)$ \\
\hline $10+$ & $1.95^{\star}$ (1.45 to 2.61$)$ & 1.65 (1.19 to 2.29$)$ \\
\hline \multicolumn{3}{|l|}{ Frailty components } \\
\hline $\begin{array}{l}\text { Low physical } \\
\text { activity }\end{array}$ & $1.41^{*}(1.15$ to 1.71$)$ & 1.22 (0.98 to 1.51$)$ \\
\hline Weight loss & 1.25 (0.94 to 1.66$)$ & 1.19 (0.89 to 1.60$)$ \\
\hline Weakness & $2.23^{*}$ (1.77 to 2.83$)$ & $1.13(0.86$ to 1.49$)$ \\
\hline Slowness & $1.99^{\star}(1.62$ to 2.45$)$ & $1.42^{*}(1.12$ to 1.81$)$ \\
\hline Exhaustion & $1.10(0.90$ to 1.35$)$ & 0.98 (0.79 to 1.22$)$ \\
\hline
\end{tabular}

${ }^{*} \mathrm{P}<0.05$.

†Adjusted for age, sex, MMSE, care home residence and comorbidities (heart attack, diabetes mellitus, bronchitis, stroke, arthritis, asthma, angina pectoris, hypertension, epilepsy, thyroid problems, Parkinson's disease, pernicious anaemia and depression).

MMSE, Mini-Mental State Examination; PIM, potentially inappropriate medication; PPI, proton pump inhibitor.

were associated with increased risk of mortality from non-frail state but not from prefrail or frail state. ${ }^{54}$

\section{Strengths and limitations of the study}

Our results were drawn from a large, nationally representative, population-based cohort study with data on both prescribed and over-the-counter medications. The strength of this analysis lies in the mutually exclusive categorisation of exposures, including polypharmacy that excluded individual PIM exposure, since the probability of PIM exposure is a function of the total number of medications. However, we did not consider medication dose or duration of use. Although we did not directly incorporate varying medication exposure as only a subset of participants underwent a single second assessment at 2 years, we did find that the exposure was typically longterm as the majority of participants who reported each PIM use at baseline, also reported the same PIM at 2-year follow-up. This suggests that much PIM use recorded in our study was 'prevalent use', reflecting long-term use among a population who are stable on each medication, and so might not be applicable to those considering a new prescription of a PIM and who may be expected to be at higher initial risk.

We could not operationalise phenotypic frailty measures exactly as per their original definition, ${ }^{5}$ although our frailty measure did independently predict mortality in this sample and our approach to measuring frailty using existing data is similar to that taken by many other epidemiological studies.

Finally, although the analysis adjusted for multiple covariates, the possibility of residual confounding remains. In particular, we could not control for every possible indication for medications contributing to polypharmacy owing to the limitations of the available data. For example, CFAS II did not record data on chronic kidney disease or heart failure that are also linked to medication use and mortality. However, controlling for the frailty variables, which reflect general health status, did not attenuate the associations between polypharmacy and death.

\section{Implications}

There are few prescribing guidelines addressing the specific needs of patients living with dementia and multimorbidity, the findings from this study will assist in understanding the safety of medicines in this population. Given these findings, consideration of the number of medications used rather than individual PIM (with the exception of antipsychotics) may be more important among cognitively impaired older adults. With respect to antipsychotics, deprescribing guidelines for behavioural and psychological symptoms of dementia have been developed and could help in reducing the harm associated with inappropriate prescribing. ${ }^{22}$

\section{Implications for the measurement and clinical application of frailty measures}

This study used a widely applied physical phenotypic definition of frailty. ${ }^{5}$ However, this definition does not account for cognitive, psychological and social frailty components, potentially resulting in underestimation of frailty in a cognitively impaired sample. ${ }^{55}$ There are no single established means of defining frailty but including frailty assessment in clinical practice is increasing. ${ }^{10}$ Slow gait speed was the only frailty marker independently associated with mortality in this study, suggesting this alone may provide as useful an assessment of frailty as the full phenotypic measure in this group. A valid, feasible and reliable frailty assessment is needed and important for identifying vulnerable individuals, applying evidencebased interventions and comparing or predicting health outcomes within this growing older population.

Frailty itself has been shown to be a strong predictor of adverse outcomes but this effect may differ with varying levels polypharmacy. ${ }^{56}$ Frailty as applied in our study did not identify those at most risk of the adverse effects of PIM. That the interaction between polypharmacy and frailty was of the opposite direction to that expected, with 
the least frail at highest relative risk calls into question the concept of frailty as reflecting susceptibility to other risks; this should be more rigorously tested in future studies.

To conclude, in this analysis of a large, nationally representative cognitively impaired sample, we found that with the exception of antipsychotics, potentially inappropriate central nervous system acting medications were not individually associated with mortality. Polypharmacy and hyperpolypharmacy were associated with an increased risk of mortality indicating that managing the number of medications used remains a priority, particularly in cognitively impaired older adults, irrespective of physical frailty.

Acknowledgements We would like to thank all CFAS II participants for their time and participation, and thank Carlotta Grossi Sampedro and Kathryn Richardson, of the University of East Anglia, for sharing the CFAS II medication codes and other guidance.

Contributors AA, BP and GMS conceived and designed the study. BP conducted the analysis and drafted the manuscript. The Cognitive Function and Ageing Study collaboration devised and conducted the CFAS II study and provided data for the current analysis. All authors were responsible for interpretation of the results, critical revision and approval of the final manuscript.

Funding This work was supported by the Alzheimer's Society (Grant Ref: AS-DTC-2014-027).

Disclaimer The funder had no input into the design, collection of data, analysis or interpretation of data nor the writing of the report or the decision to submit for publication.

Competing interests None declared.

Patient consent for publication Not required.

Provenance and peer review Not commissioned; externally peer reviewed.

Data sharing statement Data from CFAS II is available to researchers on the application as per the process described at www.cfas.ac.uk.

Open access This is an open access article distributed in accordance with the Creative Commons Attribution Non Commercial (CC BY-NC 4.0) license, which permits others to distribute, remix, adapt, build upon this work non-commercially, and license their derivative works on different terms, provided the original work is properly cited, appropriate credit is given, any changes made indicated, and the use is non-commercial. See: http://creativecommons.org/licenses/by-nc/4.0/.

\section{REFERENCES}

1. Alzheimer's Society. Dementia UK: update. 2014 https://www. alzheimers.org.uk/about-us/policy-and-influencing/dementia-ukreport

2. All-Party Parliamentary Group. Dementia rarely travels alone, 2016. https://www.alzheimers.org.uk/appg

3. Fried TR, O'Leary J, Towle V, et al. Health outcomes associated with polypharmacy in community-dwelling older adults: a systematic review. J Am Geriatr Soc 2014;62:2261-72.

4. Veronese N, Stubbs B, Noale M, et al. Polypharmacy is associated with higher frailty risk in older people: an 8-year longitudinal cohort study. J Am Med Dir Assoc 2017;18:624-8.

5. Fried LP, Tangen CM, Walston J, et al. Frailty in older adults: evidence for a phenotype. J Gerontol A Biol Sci Med Sci 2001;56:M146-M157.

6. Rockwood K, Song X, MacKnight C, et al. A global clinical measure of fitness and frailty in elderly people. CMAJ 2005;173:489-95.

7. Clegg A, Young J, lliffe S, et al. Frailty in elderly people. Lancet 2013;381:752-62.

8. Theou O, Sluggett JK, Bell JS, et al. Frailty, hospitalization, and mortality in residential aged care. J Gerontol A Biol Sci Med Sci 2018;73:1-7.

9. Dani M, Owen LH, Jackson TA, et al. Delirium, frailty, and mortality: interactions in a prospective study of hospitalized older people. $J$ Gerontol A Biol Sci Med Sci 2018;73:415-8.

10. NHS England. Updated guidance on supporting routine frailty identification and frailty care through the GP Contract 2017/2018, 2017.
11. Bonaga B, Sánchez-Jurado PM, Martínez-Reig M, et al. Frailty, polypharmacy, and health outcomes in older adults: the frailty and dependence in albacete study. J Am Med Dir Assoc 2018;19:46-52.

12. Sköldunger A, Fastbom J, Wimo A, et al. Impact of inappropriate drug use on hospitalizations, mortality, and costs in older persons and persons with dementia: findings from the SNAC study. Drugs Aging 2015;32:671-8.

13. Renom-Guiteras A, Thürmann PA, Miralles R, et al. Potentially inappropriate medication among people with dementia in eight European countries. Age Ageing 2018:47:68-74.

14. Dedhiya SD, Hancock E, Craig BA, et al. Incident use and outcomes associated with potentially inappropriate medication use in older adults. Am J Geriatr Pharmacother 2010;8:562-70.

15. Bradley MC, Motterlini N, Padmanabhan S, et al. Potentially inappropriate prescribing among older people in the United Kingdom. BMC Geriatr 2014;14:72.

16. Barry HE, Cooper JA, Ryan C, et al. Potentially inappropriate prescribing among people with dementia in primary care: a retrospective cross-sectional study using the enhanced prescribing database. J Alzheimers Dis 2016;52:1503-13.

17. Fick DM, Selma DM, Beizer DM, et al. American Geriatrics Society 2015 updated beers criteria for potentially inappropriate medication use in older adults. J Am Geriatr Soc 2015;11:2227-46.

18. O'Mahony D, O'Sullivan D, Byrne S, et al. STOPP/START criteria for potentially inappropriate prescribing in older people: version 2. Age Ageing 2015;44:213-8.

19. Schoenfeld AJ, Grady D. Adverse effects associated with proton pump inhibitors. JAMA Intern Med 2016;176:172-4.

20. Eom CS, Jeon CY, Lim JW, et al. Use of acid-suppressive drugs and risk of pneumonia: a systematic review and meta-analysis. CMAJ 2011;183:310-9.

21. Filion KB, Chateau D, Targownik LE, et al. Proton pump inhibitors and the risk of hospitalisation for community-acquired pneumonia: replicated cohort studies with meta-analysis. Gut 2014;63:552-8.

22. Bjerre LM, Ramsay T, Cahir C, et al. Assessing potentially inappropriate prescribing (PIP) and predicting patient outcomes in Ontario's older population: a population-based cohort study applying subsets of the STOPP/START and Beers' criteria in large health administrative databases. BMJ Open 2015;5:e010146.

23. Cunningham R, Dale $B$, Undy $B$, et al. Proton pump inhibitors as a risk factor for Clostridium difficile diarrhoea. $J$ Hosp Infect 2003;54:243-5.

24. Kwok CS, Arthur AK, Anibueze Cl, et al. Risk of Clostridium difficile infection with acid suppressing drugs and antibiotics: meta-analysis. Am J Gastroenterol 2012;107:1011-9.

25. Yang YX, Lewis JD, Epstein S, et al. Long-term proton pump inhibitor therapy and risk of hip fracture. JAMA 2006;296:2947-53.

26. Ngamruengphong S, Leontiadis Gl, Radhi S, et al. Proton pump inhibitors and risk of fracture: a systematic review and meta-analysis of observational studies. Am J Gastroenterol 2011;106:1209-18.

27. Othman F, Card TR, Crooks CJ. Proton pump inhibitor prescribing patterns in the UK: a primary care database study. Pharmacoepidemiol Drug Saf 2016;25:1079-87.

28. British National Formulary. Antipsychotic drugs. 2011 https://bnf. nice.org.uk/drug-class/antipsychotic-drugs.html.

29. McEvoy RD, Nyfort-Hansen KS. Sleep disorders in the elderly: the pros and cons of prescribing. In: Prescribing for Elderly Patients. Chichester, UK: John Wiley \& Sons, Ltd:45-52.

30. Alexopoulos GS, Streim J, Carpenter D, et al. Expert Consensus Panel for Using Antipsychotic Drugs in Older Patients. Using antipsychotic agents in older patients. J Clin Psychiatry 2004;65 Suppl 2:5-99.

31. Glass J, Lanctôt KL, Herrmann N, et al. Sedative hypnotics in older people with insomnia: meta-analysis of risks and benefits. BMJ 2005;331:1169.

32. Mintzer J, Burns A. Anticholinergic side-effects of drugs in elderly people. J R Soc Med 2000;93:457-62.

33. Chatterjee S, Bali V, Carnahan RM, et al. Risk of mortality associated with anticholinergic use in elderly nursing home residents with depression. Drugs Aging 2017;34:691-700.

34. Mehta DC, Short JL, Hilmer SN, et al. Drug access to the central nervous system in Alzheimer's disease: preclinical and clinical insights. Pharm Res 2015;32:819-39.

35. Reeve E, Trenaman SC, Rockwood K, et al. Pharmacokinetic and pharmacodynamic alterations in older people with dementia. Expert Opin Drug Metab Toxicol 2017;13:651-68.

36. Mallet L, Spinewine A, Huang A. The challenge of managing drug interactions in elderly people. Lancet 2007;370:185-91.

37. Matthews FE, Arthur A, Barnes LE, et al. A two-decade comparison of prevalence of dementia in individuals aged 65 years and older from three geographical areas of England: results 
of the Cognitive Function and Ageing Study I and II. Lancet 2013;382:1405-12.

38. Matthews FE, Stephan BC, McKeith IG, et al. Two-year progression from mild cognitive impairment to dementia: to what extent do different definitions agree? J Am Geriatr Soc 2008;56:1424-33.

39. Booth N. What are the Read Codes? Health Libr Rev 1994;11:177-82.

40. Farrell B, Tsang C, Raman-Wilms L, et al. What are priorities for deprescribing for elderly patients? Capturing the voice of practitioners: a modified delphi process. PLoS One 2015;10:e0122246.

41. Boustani M, Campbell N, Munger S, et al. Impact of anticholinergics on the aging brain: a review and practical application. Aging health 2008;4:311-20.

42. Herr $\mathrm{M}$, Robine JM, Pinot $\mathrm{J}$, et al. Polypharmacy and frailty: prevalence, relationship, and impact on mortality in a French sample of 2350 old people. Pharmacoepidemiol Drug Saf 2015;24:637-46.

43. Dent E, Kowal P, Hoogendijk EO. Frailty measurement in research and clinical practice: A review. Eur J Intern Med 2016;31:3-10.

44. Savva GM, Donoghue OA, Horgan F, et al. Using timed up-and-go to identify frail members of the older population. $J$ Gerontol A Biol Sci Med Sci 2013;68:441-6.

45. Gale CR, Cooper C, Sayer AA. Prevalence of frailty and disability: findings from the English Longitudinal Study of Ageing. Age Ageing 2015;44:162-5.

46. McCarthy EK, Horvat MA, Holtsberg PA, et al. Repeated chair stands as a measure of lower limb strength in sexagenarian women. $J$ Gerontol A Biol Sci Med Sci 2004;59:1207-12.
47. Schoenfeld D. Partial residuals for the proportional hazards regression model. Biometrika 1982;69:239-41.

48. Douglas IJ, Smeeth L. Exposure to antipsychotics and risk of stroke: self controlled case series study. BMJ 2008;337:a1227.

49. Cross AJ, George J, Woodward MC, et al. Potentially Inappropriate Medication, Anticholinergic Burden, and Mortality in People Attending Memory Clinics. J Alzheimers Dis 2017;60:349-58.

50. Richardson K, Fox C, Maidment I, et al. Anticholinergic drugs and risk of dementia: case-control study. BMJ 2018;361:k1315.

51. Molloy D, Molloy A, O'Loughlin C, et al. Inappropriate use of proton pump inhibitors. Ir J Med Sci 2010;179:73-5.

52. Haastrup P, Paulsen MS, Zwisler JE, et al. Rapidly increasing prescribing of proton pump inhibitors in primary care despite interventions: a nationwide observational study. Eur J Gen Pract 2014;20:290-3.

53. Schoenfeld AJ, Grady D. Adverse effects associated with proton pump inhibitors. JAMA Intern Med 2016;176:172.

54. Jamsen KM, Bell JS, Hilmer SN, et al. Effects of changes in number of medications and drug burden index exposure on transitions between frailty states and death: the concord health and ageing in men project cohort study. J Am Geriatr Soc 2016;64:89-95.

55. Collard RM, Boter H, Schoevers RA, et al. Prevalence of frailty in community-dwelling older persons: a systematic review. J Am Geriatr Soc 2012;60:1487-92.

56. Kojima G, Iliffe S, Walters K. Frailty index as a predictor of mortality: a systematic review and meta-analysis. Age Ageing 2018;47:193-200. 